\title{
Well-known Theorems on the Ellipse deduced by Projection from the Circle; also a Theorem on Curvature.
}

\author{
By Robert J. T. BeLL.
}

1. In the treatment of the geometry of the ellipse, projection from the circle offers an easy and direct means of obtaining the properties of conjugate diameters of the ellipse. In text-books generally, little further use is made of the method of projection, except in the case of a few exercises. The following note shows how it can be employed to derive most of the well-known properties of the ellipse.

2. We shall use the notation p. $A B$ for "the projection of $A B$," and $p .{ }^{2} A B$ for "the square on the projection of $A B$," and employ the following two theorems:

I. If we have two planes $P$ and $Q$, inclined at an angle $\alpha$ and intersecting in a line $O X$, and if rectangular axes are taken in each plane with $O X$ as a common $x$-axis, then if $m$ is the gradient of any line in $P$ and $m^{\prime}$ is the gradient of its projection on $Q, m^{\prime}=m \cos \alpha$.

II. If a segment of length $r$ in the plane $P$ makes an angle $\theta$ with $O X$, the length of its projection on the plane $Q$ is $r \sqrt{1-\sin ^{2} \alpha \sin { }^{2} \theta}$. These are proved from the usual figure:-

(i)

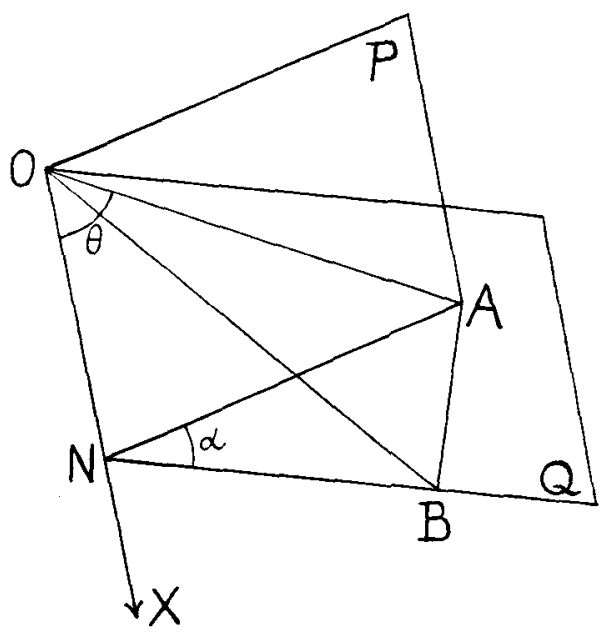

$$
\begin{aligned}
N A & =m \cdot O N, \\
N B & =m^{\prime} \cdot O N . \\
\therefore \quad \text { and } \quad N B & =N A \cos \alpha, \\
m^{\prime} & =m \cos \alpha .
\end{aligned}
$$


xiv

$$
\begin{aligned}
O B^{2} & =O N^{2}+N B^{2}, \\
& =O A^{2} \cos ^{2} \theta+O A^{2} \sin ^{2} \theta \cos ^{2} \alpha, \\
& =r^{2}\left(1-\sin ^{2} \theta \sin ^{2} \alpha\right) .
\end{aligned}
$$

3. To obtain the ellipse $\frac{x^{2}}{a^{2}}+\frac{y^{2}}{b^{2}}=1$ from the circle $x^{2}+y^{2}=a^{2}$, we rotate the circle about the $x$-axis until its plane makes an angle $a=\cos ^{-1}\left(\frac{b}{a}\right)$ with the original plane, and then project it on to the original plane. The eccentricity of the ellipse is $\sin a$, and the foci, $S$ and $S^{\prime}$, are $( \pm a \sin a, 0)$.

From I. it at once follows that

(i) the product of the gradients of conjugate diameters is $-b^{2} / a^{2}$;

(ii) the gradients of the equal conjugate diameters, i.e., the projections of the bisectors of the angles between the axes, are $\pm b / a$.

4. If $P$ is any point on the circle, $C$ being the centre and $S$ and $S^{\prime}$ the points $( \pm a \sin \alpha, 0)$, we can easily find the projections of $S P$, $S^{\prime} P, C P$.

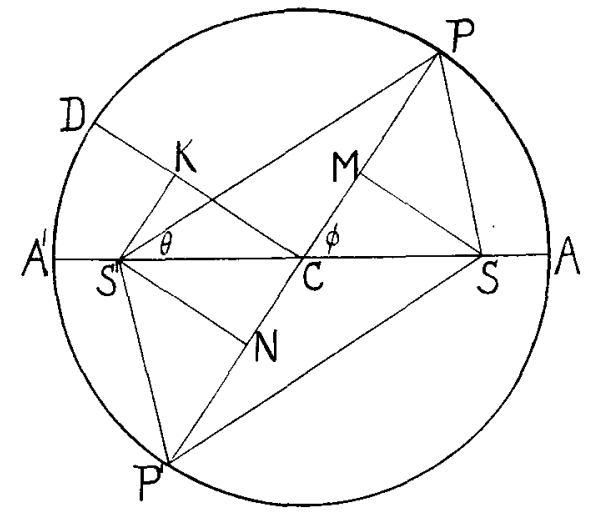

Produce $P C$ to cut the circle in $P^{\prime}$ and draw $S^{\prime} N$ and $S M$ at right angles to $P P^{\prime}$. Let the angles $P S^{\prime} C, P C S$ be $\theta$ and $\phi$. Then from the triangle $C P S^{\prime}, \frac{\sin C P S^{\prime}}{C S^{\prime}}=\frac{\sin \theta}{a}$, and $C S^{\prime}=a \sin \alpha$.

$$
\therefore \quad \sin C P S^{\prime}=\sin \theta \sin \alpha \text {. }
$$

The projection of $S^{\prime} P=S^{\prime} P \sqrt{1-\sin ^{2} \theta \sin ^{2} \alpha}$

$$
\begin{aligned}
& =S^{\prime} P \cos C P S^{\prime} \\
& =N P .
\end{aligned}
$$

Again $\quad$ p. $S P=p \cdot P^{\prime} S^{\prime}=P^{\prime} N$, in the same way.

Further, since $S M=C S \sin \phi=a \sin a \sin \phi$,

$$
p .^{2} C P=C P^{2}-S M^{2} \text {. }
$$


Hence we have

(i) $p . S^{\prime} P+p . S P=P N+N P^{\prime}=2 a$, i.e. in the ellipse $S P+S^{\prime} P=2 a$;

(ii) if $C P$ and $C D$ are perpendicular radii,

$$
\begin{aligned}
p \cdot{ }^{2} C P+p .{ }^{2} C D & =\left(C P^{2}-S M^{2}\right)+\left(C D^{2}-S^{\prime} K^{2}\right), \\
& =2 a^{2}-C S^{2}=2 a^{2}-a^{2} \sin ^{2} a=a^{2}+b^{2},
\end{aligned}
$$

i.e. the sum of the squares on two conjugate semi-diameters is constant and $=a^{2}+b^{2}$.

(iii) $(p . S P)\left(p . S^{\prime} P\right)=P M . M P^{\prime}=C P^{2}-C M^{2}=C D^{2}-S^{\prime} K^{2}=p .{ }^{2} C D$, i.e. in the ellipse $S P . S^{\prime} P=C D^{2}$.

5. Another consequence of Theorem I. is that the projections on the plane of the ellipse of two lines in the plane of the circle, such that the product of their gradients is $-a^{2} / b^{2}$, are perpendicular lines.

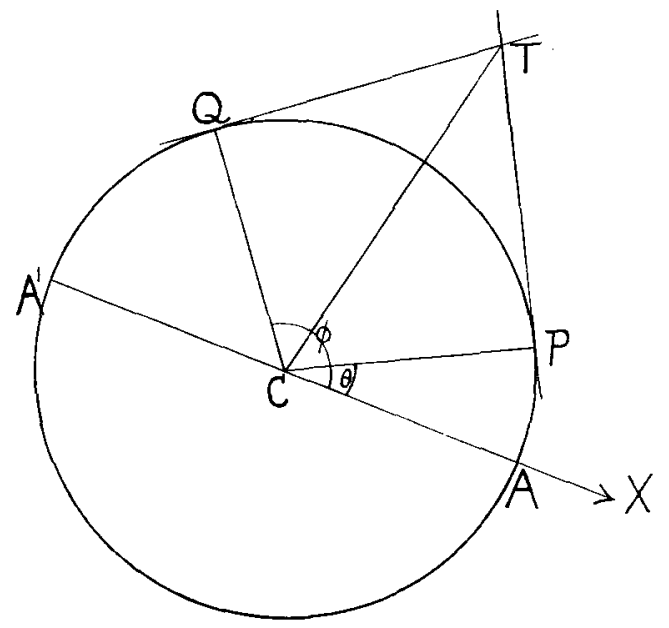

Suppose that the tangents from $T, T P$ and $T Q$, are such lines. Then if $C P, C Q$ make angles $\theta$ and $\phi$ with $C X, \tan \theta \tan \phi=-\frac{b^{2}}{a^{2}}$.

Now

$$
C T=C P \sec \frac{\phi-\theta}{2},
$$

$\therefore \quad \quad \quad p .{ }^{2} C T=C T^{2}\left(1-\sin ^{2} \alpha \sin ^{2} \frac{\phi+\theta}{2}\right)$,

$$
=\frac{a^{2}}{\cos ^{2} \frac{\phi-\theta}{2}}\left(1-\frac{a^{2}-b^{2}}{a^{2}} \sin ^{2} \frac{\phi+\theta}{2}\right) .
$$

$\mathrm{But} \frac{\cos \phi \cos \theta}{a^{2}}=\frac{\sin \phi \sin \theta}{-b^{2}}=-\frac{1-2 \sin ^{2} \frac{\phi+\theta}{2}}{a^{2}+b^{2}}=\frac{2 \cos ^{2} \frac{\phi}{2}-\theta}{a^{2}-b^{2}}$. 
xvi

$\therefore \quad a^{2}-\left(a^{2}-b^{2}\right) \sin ^{2} \frac{\phi+\theta}{2}=\left(a^{2}+b^{2}\right) \cos ^{2} \frac{\phi-\theta}{2}$,

$\therefore \quad \quad p .^{2} C T=a^{2}+b^{2}$.

$\therefore$ the locus of the point of intersection of perpendicular tangents to the ellipse is the circle whose centre is $C$ and radius $\sqrt{a^{2}+b^{2}}$.

6. We shall now obtain some properties of the focus and directrix.

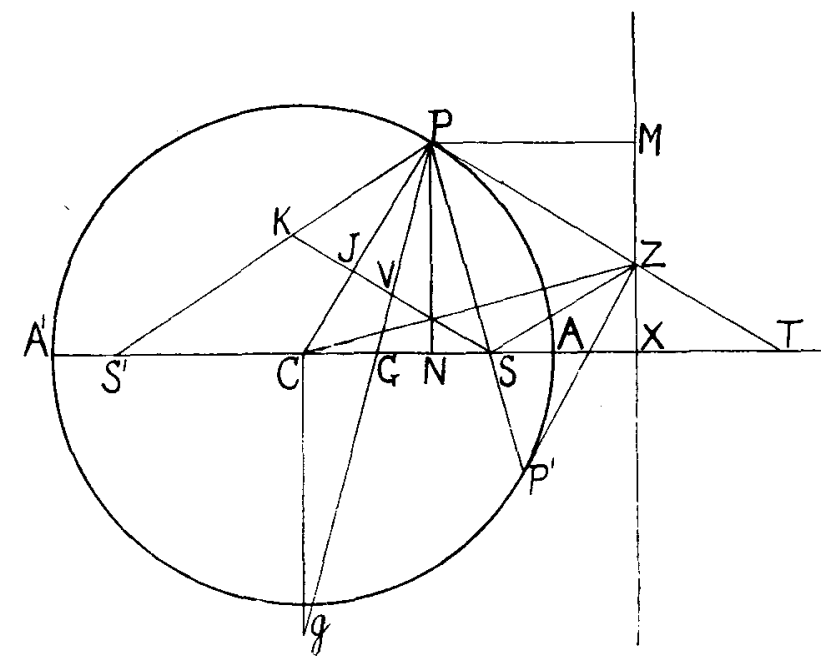

Let $X Z$ be the polar of $S$ w.r.t. the circle, and let the tangent at any point $P$ on the circle meet $X Z$ in $Z$. Then $C S=a e=a \sin a$, and $C X=a / e=a \operatorname{cosec} \alpha$. Draw $P N, S J$ at right angles to $C A$ and $C P$. Then if $C N=x, C P . C J=C S . C N, \therefore C J=e x$.

Hence $p . S P=J P($ by $\S 4)=C P-C J=a-e x$

$$
=e(a / e-x)=e(C X-C N)=e N X=e P M .
$$

$\therefore$ for the ellipse, $X Z M$ projects into a directrix for which we have the property $S P=e P M$.

Again $P S P^{\prime}$ is the polar of $Z$ w.r.t. the circle and $P^{\prime} Z$ is the tangent at $P^{\prime}$. Hence for the ellipse, tangents at the ends of a focal chord intersect on the directrix. Further the reciprocal of the product of the gradients of $S P$ and $S Z$

$$
\begin{aligned}
& =-\frac{X Z}{C X} \cdot \frac{S X}{X Z},(\text { since } C Z \perp S P) . \\
& =-\frac{a / e-a e}{a / e}=-\left(1-e^{2}\right)=-\cos ^{2} \alpha .
\end{aligned}
$$

$\therefore \quad S Z$ and $S P$ project into perpendicular lines (by I.),

$\therefore \quad$ in the ellipse, $P Z$ subtends a right angle at the focus. 
7. If we bisect $S K$ at $V$ and produce $P V$ to meet $A^{\prime} A$ in $G$, then since $S K \| P T, P T, P S, P G$ and $P S^{\prime}$ form a harmonic pencil and $\left(S^{\prime} S, G T\right)$ is a $H . R$. Now the reciprocal of the product of the gradients of $P T$ and $P G$

$$
=-\frac{\text { gradient of } C P}{\text { gradient of } P G}=-\frac{N P}{C N} \cdot \frac{G N}{N P}=-\frac{G N}{C N} .
$$

But $C N . C T=C A^{2}$ and $C G . C T=C S^{2}, \therefore e^{2} C N=C G$.

$$
\therefore \quad \frac{G N}{C N}=\frac{C N-C G}{C N}=1-e^{2}=\cos ^{2} \alpha .
$$

$\therefore P G$ and $P T$ project into perpendicular lines. Hence in the ellipse the tangent and the normal at $P$ bisect the angles between $S P$ and $S^{\prime} P$.

Again we have found that $C G=e^{2} C N$ and that $J P=a-e C N$.

$\therefore \quad G S=C S-C G=a e-e^{-2} x=e(a-e x)=e . J P=e(p . S P)$.

$\therefore$ in the ellipse, $G S=e . S P$.

Further, if $P G$ is produced to meet the perpendicular through $C$ to $A^{\prime} A$ in $g$, we have, from the similar triangles $P G N, g G C$, $\frac{P G}{P g}=\frac{G N}{C N}=1-e^{2}=\frac{b^{2}}{a^{2}}$.

Thus in the ellipse if the normal at $P$ meets the axes in $G$ and $g$, $\frac{P G}{P g}=$ constant $=\frac{b^{2}}{a^{2}}$.

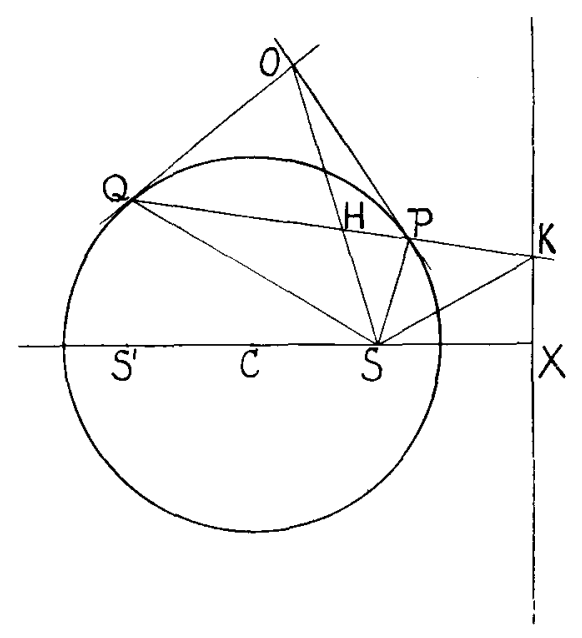

8. If the chord of contact of the tangents from any point $O$ meets the polar of $S$ in $K, O S$ is the polar of $K$ and $(K H, P Q)$ is a harmonic 


\section{xviii}

range. It can be shown, as in the last paragraph, that the product of the gradients of $S K$ and $S O$ is $-a^{2} / b^{2}$ and hence the projections of $S K$ and $S O$ are perpendicular rays of a harmonic pencil. Therefore in the ellipse, two tangents $O P$ and $O Q$ subtend equal angles at a focus.

9. It follows immediately, by projection from the circle, that if any line through a fixed point $O$ cut an ellipse in $P$ and $Q$, and if $C R$ is the semidiameter parallel to $O P Q$, then the ratio $O P . O Q: C R^{2}$ is constant. Hence if any circle cuts the ellipse in $P, Q, P^{\prime}, Q^{\prime}$, the semidiameters parallel to $P Q$ and $P^{\prime} Q^{\prime}$ are equal and so they, and therefore $P Q$ and $P^{\prime} Q^{\prime}$, are equally inclined to the axes. As a special case, we have that the tangent at $P$ and the common chord of the ellipse and its circle of curvature at $P$ are equally inclined to the axes.

10. From this we obtain the following theorem, which gives a geometrical construction for the centre of curvature, and an easy method of finding the radius of curvature and the coordinates of the centre:-

If $\omega$ is the centre of curvature at a point $P$ on the ellipse and $P \omega$ meets the major and minor axes in $G$ and $g$ respectively, and if through $G$ and $g$ parallels to the tangent at $P$ are drawn to cut $C P$ in $\nu$ and $\nu^{\prime}$ respectively, then $\omega \nu$ and $\omega \nu^{\prime}$ are parallel to the axes.

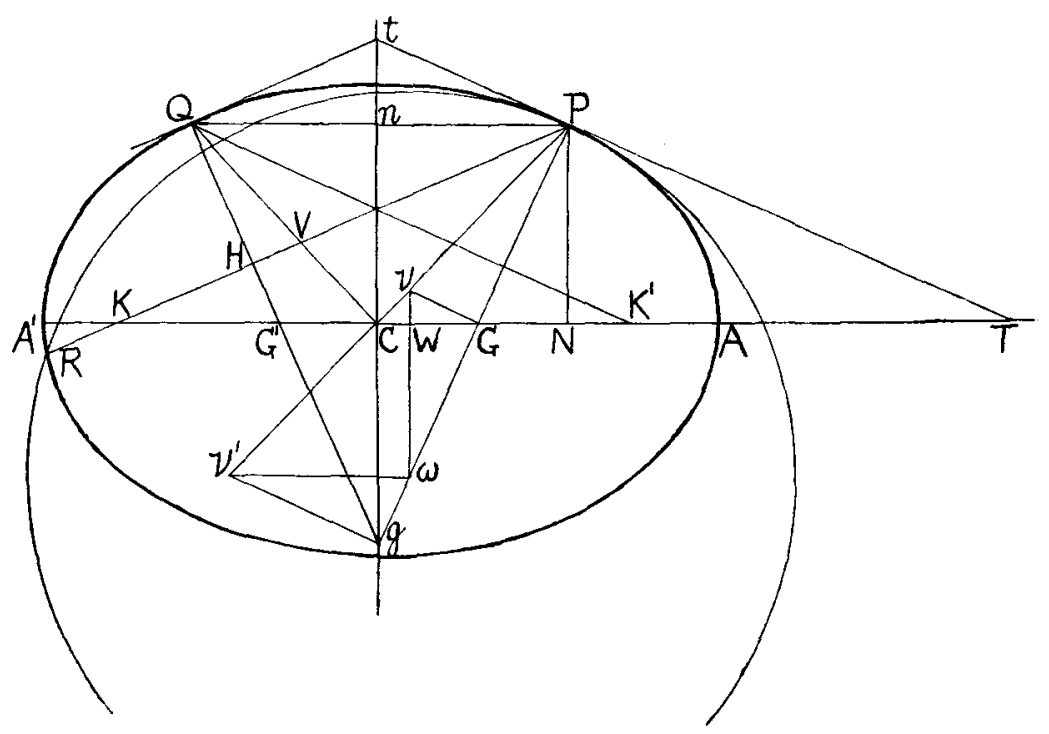


For if $P Q$, parallel to the major axis, cuts the ellipse in $Q, P R$, the chord of curvature through $P$, is parallel to the tangent at $Q$ and therefore $C Q$ bisects $P R$ at $V . Q g$ is the normal at $Q$,

$\therefore \quad Q g \perp P R$ and $\| V \omega$.

From similar triangles, $\frac{P H}{H K}=\frac{P Q}{K G^{\prime}} ; \therefore \frac{P H}{P K}=\frac{P Q}{P Q+K G^{\prime}}=\frac{P Q}{G T}$.

And similarly

$$
\frac{P V}{P K}=\frac{P Q}{C T}
$$

$$
\begin{array}{ll}
\therefore & \frac{P g}{P \omega}=\frac{P H}{P V}=\frac{C T}{G T}=\frac{P C}{P \nu}, \\
\therefore & \nu \omega \| C g .
\end{array}
$$

Again $\quad \frac{P G}{P g}=\frac{P \nu}{P \nu^{\prime}}, \therefore \frac{P G}{P \omega}=\frac{P C}{P \nu^{\prime}}, \therefore \nu^{\prime} \omega \| C G$.

From $\S 7$, we have that $\frac{P G}{P g}=\frac{b^{2}}{a^{2}}$,

$$
\therefore \quad g n=\frac{a^{2}}{b^{2}} \cdot N P=\frac{a^{2} \sin \theta}{b} ; \text { and } n P=a \cos \theta,
$$

$\therefore \quad P g^{2}=\frac{a^{2}}{b^{2}}\left(a^{-2} \sin ^{2} \theta+b^{2} \cos ^{2} \theta\right)=\frac{a^{2}}{b^{2}} C D^{2}$, where $C D$ is the semidiameter conjugate to $C P$.

$$
\begin{gathered}
\text { But } \frac{T P^{2}}{T A \cdot T^{\prime}}=\frac{C D^{2}}{C A^{2}}, T P^{2}=T N . T G \text { and } T A . T A^{\prime}=C T^{2}-C A^{2} \\
=C T^{\circ}-C T \cdot C N=C T \cdot N T . \\
\therefore \quad \frac{P \omega}{P g}=\frac{P \nu}{P C}=\frac{T G}{T C}=\frac{C D^{2}}{C A^{2}}, \text { and } \therefore P \omega=\frac{C D^{3}}{a b} .
\end{gathered}
$$

Now since $C G . C T=C S^{2}$ and $C T=\frac{a^{2}}{x}=a \sec \theta$,

$$
\begin{gathered}
\frac{C W}{C N}=\frac{C v}{C P}=\frac{C G}{C T}=\frac{a e^{2} \cos \theta}{a \sec \theta}=e^{2} \cos ^{2} \theta, \\
\therefore \quad C W=a e^{2} \cos ^{3} \theta .
\end{gathered}
$$

Again $\frac{W \omega}{C g}=\frac{C G-C W}{C G}=1-\frac{C W}{C G}=1-\cos ^{2} \theta=\sin ^{2} \theta$ 


$$
\begin{gathered}
\text { and } C g=n g-n C=-\frac{a^{2} \sin \theta}{b}+b \sin \theta=-\frac{a^{2} e^{2} \sin \theta}{b}, \\
\therefore \quad W \omega=-\frac{a^{2} e^{-2} \sin ^{3} \theta}{b} .
\end{gathered}
$$

Hence the centre of curvature is $\left(\frac{a^{2} e^{2} \cos ^{3} \theta}{a},-\frac{a^{2} e^{2} \sin ^{3} \theta}{b}\right)$.

\title{
The "Pellian Equation" and Some Series for $\pi$.
}

\author{
By A. C. Attken.
}

$\S 1$. The craze for extensive $\pi$-calculation which was so strange a feature of the last century was probably brought to an end not so much by the famous 707 decimals of W. Shanks in 1873 as by the demonstrations of Hermite and Lindemann, about the same time, regarding the transcendental nature of both $e$ and $\pi$. Sporadic minor outbreaks of the disease still occur, of course, -Ramanujan in his earlier days was not entirely immune-and the series of the present note may seem symptomatic. It is hoped, however, that they will not be devoid of interest from the point of view of elementary trigonometry.

$\S 2$. It is mentioned in the standard texts that a useful series for evaluating $\pi$ is Gregory's inverse tangent series,

$$
\arctan x=x-x^{3} / s+x^{5} / 5-x^{i} / ;+\ldots,
$$

but that for $x=1$ it converges to $\pi / 4$ with extreme slowness. Machin's formula,

$$
\arctan 1=4 \arctan \frac{1}{\overline{5}}-\arctan \frac{1}{239},
$$

and variants such as Rutherford's give a greatly enhanced convergency.

The reason why the corresponding series for $\pi / 6, \pi / 8, \pi / 10, \pi / 12$ have not found favour is an obvious one; the tangents of these angles are irrational, $\tan \pi / 10$ even involving a double surd.

Consider however, for example, the sequence of rational approxi- 